Cultures \& Conflits

40 | hiver 2000

Pacifications. Réconciliations (1)

\title{
"Le pardon est un acte de volonté". Entretien avec Anthony Holliday
}

\section{Anthony Holiday}

\section{(2) OpenEdition}

1 Journals

\section{Édition électronique}

URL : http://journals.openedition.org/conflits/477

DOI : $10.4000 /$ conflits. 477

ISSN : $1777-5345$

Éditeur :

CCLS - Centre d'études sur les conflits lilberté et sécurité, L'Harmattan

\section{Édition imprimée}

Date de publication : 1 septembre 2000

ISBN : 2-7475-0572-3

ISSN : 1157-996X

\section{Référence électronique}

Anthony Holiday, «"Le pardon est un acte de volonté". Entretien avec Anthony Holliday », Cultures \& Conflits [En ligne], 40 | hiver 2000, mis en ligne le 28 septembre 2006, consulté le 30 mars 2021. URL http://journals.openedition.org/conflits/477 ; DOI : https://doi.org/10.4000/conflits.477

Ce document a été généré automatiquement le 30 mars 2021.

Creative Commons License 


\title{
"Le pardon est un acte de volonté". Entretien avec Anthony Holliday
}

\author{
Anthony Holiday
}

« Le pardon est un acte de volonté » : entretien avec Anthony Holiday ${ }^{1}$ Pensez-vous que le nouveau régime démocratique de votre pays soit stable ? Il est encore trop tôt pour le dire avec certitude. Le régime est en tout cas plus stable que nom-bre de ses homologues africains. Le facteur essentiel de stabilité à moyen terme est la gou-vernance. Il y a des signes encourageants, qui suggèrent que le Parlement commence à s'af-fir-mer comme critique et conseiller de la présidence: par exemple dans les réactions des com-mis-sions parlementaires aux décisions du gouvernement, aux projets de loi, etc.; ainsi que dans sa réponse étonnamment vigoureuse au récent scandale sur les achats d'armements. Néan-moins, la situation générale est inquiétante. $\mathrm{Du}$ point de vue constitutionnel, la présidence sud-africaine est incontestablement « exé-cu-tive »: le Président a plus de pouvoir, et subit moins de contraintes, que, par exemple, le Pré-sident des États-Unis ou le Premier Ministre britannique. Même eu égard à ses pré-ro-ga-tives, cependant, le Président Mbeki a adopté un style de direction que, à son grand dépit, j’ai appelé « léniniste » dans un article de journal. Il a fusionné dans un même bureau la pré-sidence et la vice-présidence, avec un cabinet de plus de 500 personnes. En association avec le Président, son Ministre sans portefeuille Essap Pahad exerce un contrôle exclusif sur les com-munications gouvernementales. Une instance spéciale, qui ne rend de comptes à per-sonne, évalue les projets de loi et autres initiatives avant même qu'ils arrivent en Conseil des Ministres. Et ce Conseil, dont les membres sont, pour la plupart, des obligés du Président Mbeki, s'est révélé docile, et se comporte à la limite en chambre d'enregistrement. Cette or-ga-ni-sation avait certainement pour but de centraliser le pouvoir afin de donner l'image d'un Prési-dent « à la barre » et de stabiliser ainsi - compte tenu de l'importance des problèmes aux-quels le pays est confronté - le nouveau régime démocratique. En contrepartie, toutefois, cette centralisation concentre les regards sur le Président comme « décideuren-chef ». Le dé-ca-lage entre les attentes ainsi créées et la réalité le fait inévitablement apparaitre comme faible, vel-lé-itaire, mal informé et mal conseillé. D'où une instabilité au cœur du système de gouvernance. De surcroît, l'Afrique du Sud n'attire pas 
l'investissement étranger à un rythme compatible avec l'objectif officiel d'un taux de croissance du PNB de 6\% par an. Au contraire, il y a eu une importante émigration de travailleurs qualifiés, et le rétrécissement de la base fiscale qui en résulte fragilise le Président et ses hommes. C'est l'un des facteurs qui avive les penchants léninistes centralisateurs et dictatoriaux de certains des favoris les plus en cour. Les circonstances sociales dont il faut tenir compte pour évaluer la stabilité du régime sont étroitement liées à ces questions économiques. La criminalité, le SIDA, et d'autres facteurs po-tentiels de déstabilisation, seront plus difficiles à gérer si les rentrées fiscales sont faibles. Il s'agit là, cependant, de facteurs d'aggravation de ce qui reste avant tout un problème de gou-ver-nance. De même, les aspects strictement démocratiques de la transition viennent surtout com-pliquer l'équation. Il existe, bien entendu, une opposition au principe même du com-pro-mis démocratique, mais ce n'est pas le facteur principal qui détermine la stabilité future du ré-gime. Quelle a été l'importance, à votre avis, de la "réconciliation " dans la transition démo-cra-tique ? Elle a été de la plus haute importance. Toutefois, il ne faut surtout pas assimiler la récon-ci-li-a-tion telle qu'elle est pensée par nombre de Sud-Africains, blancs et noirs, avec la définition - le plus souvent incohérente - qui en a été donnée par des personnages publics comme Mon-seigneur Tutu, prix Nobel de la paix, et sa Commission sur la Vérité et la Réconciliation (Truth and Reconciliation Commission TRC). Comme j'ai essayé de le montrer au moment des auditions de la TRC ${ }^{2}$, le pardon traduit une forme particulière de conscience intime. Il n'a guère de rapport avec la théâtralité déplaisante - à mon sens - qui a souvent caractérisé les auditions de la TRC ; et strictement aucun rapport avec les jérémiades évangélistes, les sup-pli-cations, la piété exhibitionniste de Monseigneur Tutu dans son rôle de Président. La ré-con-ciliation, comme j'ai également essayé de le montrer, n'est pas incompatible avec le juste châ-ti-ment des maux infligés pendant la période de terreur raciste que fut l'apartheid. Pour autant qu'il y ait eu réconciliation, elle a pris une multitude de formes, souvent tacites, et a concerné les Sud-Africains les plus divers, quelles que soient leurs origines et leurs af-fi-li-a-tions. Je suis convaincu depuis longtemps que ces Sud-Africains étaient très en avance sur les hommes politiques qui cherchaient à contrôler leurs actions, y compris dans ce domaine in-ti-me-ment personnel, et dans un autre monde par rapport aux politologues, aux psy-cho-logues, aux journalistes d'opinion qui prétendaient les comprendre. On ne peut, cependant, évaluer la TRC sans tenir compte de sa double référence à la vérité et la réconciliation. Les membres de la Commission eux-mêmes ne prétendent pas avoir établi la vérité complète sur les violations des droits de l'homme sous l'apartheid. Ce n'est guère sur-prenant. Compte tenu de leur étendue, il est pratiquement impossible de les dévoiler et de les raconter d'une manière compatible avec la rigueur historiographique. Mais la TRC a égale-ment été prisonnière de ses statuts, qui lui ont imposé un mode particulier d'enquête et d'au-di-tion. En effet, il était prévu que l'amnistie soit accordée aux auteurs avoués de violations des

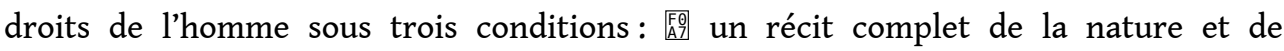
l'étendue de ces violations et des circonstances qui les ont entourées; 盾 un mobile politique avéré; 瞇 une certaine proportionnalité entre l'acte constitutif de la violation et son objectif politique affiché, selon les principes Norgaard, qui sont internationalement admis. Il en résulte que les témoignages sur lesquels la Commission devait s'appuyer, souvent de manière décisive, émanaient de ce que l'on appelle en droit sud-africain des "témoins com-plices" (accomplice witnesses), à savoir des témoins à charge qui témoignent en échange de l'abandon des poursuites à leur 
encontre. En droit sud-africain, pour des raisons évidentes, un principe strict de précaution détermine le poids qu'un juge peut attribuer à de tels té-moi-gna-ges. La TRC, au contraire, a dû s'efforcer d'établir les faits historiques à partir d'une alliance inconfortable entre cette objectivité judiciaire procédurale et les doctrines souvent confuses et déroutantes de la théologie de la libération et de la psychothérapie. Trouvez-vous que l'on puisse justifier l'usage de l'amnistie dans la processus de «récon-ci-li-ation"? Je laisserai de côté pour l'instant certains cas précis d'amnistie accordée par la TRC, qui me semblent déplorables. En termes généraux, il me paraît évident qu'il n'y a guère de sens, d'un point de vue de théorie du droit, à soutenir que l'amnistie ne peut jamais se justifier. Adopter un tel point de vue absolutiste, ce serait condamner nos juridictions pénales à un fonc-tion-ne-ment encore plus difficile que celui dont, compte tenu de la surcharge de notre justice, elles souffrent déjà. L'amnistie de certains prévenus ou suspects peut se révéler né-ces-saire, dans cer-taines circonstances, pour permettre à d'autres criminels - peut-être plus grands - d'être traduits en justice. Dans un cadre juridique normal, toutefois, un coupable avoué qui bénéficierait d'un abandon de poursuites pénales en échange de son témoignage ne pourrait, en principe, échapper à la res-ponsabilité civile devant les victimes ou leurs ayants droit. De ce point de vue, la légis-lation qui a établi la TRC a constitué une rupture radicale avec la procédure normale. Elle a en effet per-mis, en théorie et en pratique, qu'un policier tortionnaire, par exemple, fasse un récit com-plet de ses pratiques scélérates, sans exprimer la moindre parcelle de remords, et reparte en-suite libre, à l'abri de toute crainte de sanctions pénales ou de poursuites civiles. On pourrait rétorquer que le dédommagement sous l'égide d'une juridiction civile est hors su-jet s'agissant d'une personne qui a subi des dommages physiques ou psychologiques perma-nents à la suite d'une violation de ses droits fondamentaux. C'est sans doute vrai dans un sens moral abstrait et très élevé, mais j'ai du mal à admettre que quelqu'un qui, par exemple, serait actuellement incapable de gagner sa vie en raison de sévices corporels infligés par la po-lice ou les services de sécurité du régime d'apartheid se verrait contester tout droit au dé-dom-ma-ge-ment financier et symbolique (par le biais de la reconnaissance publique, par une ju-ri-diction constituée, du dommage infligé) quand cela lui faciliterait la poursuite d'une vie plus ou moins normale. Y a-t-il de choses que vous ne pouvez pas pardonner? La formulation de cette question pose problème, dans la mesure où elle semble renvoyer aux propriétés intrinsèques, ou supposées telles, de ce qu'il s'agirait de pardonner. Or, le pardon est un acte de volonté. La vraie question est, me semble-t-il: «y a-t-il des choses que je ne veux pas pardonner? ». Et, dans cette formulation, la réponse est «oui ». Je ne veux pas pardon-ner au commandant Craig Williamson le meurtre de la femme et des enfants de mon cou-sin et le meurtre de Ruth First ${ }^{3}$. Williamson a récemment été amnistié par un comité de la TRC. Cette décision, cependant, est contestée devant les tribunaux. L'affaire est en cours, et je ne souhaite donc pas, pour des raisons personnelles et juridiques que j'espère com-pré-hen-sibles, en dire davantage. Pourra-t-on, devrait-on, oublier les événements historiques les plus tragiques? Je commencerai par essayer de répondre directement à cette question difficile. Il y a un sens très clair dans lequel il ne sera pas possible d'oublier les événements de l'histoire sud-africaine que la question (à tort ou de raison) désigne comme "tragiques». Comme je le dis dans mon analyse philosophique de la question, si quelqu'un me fait du mal en amputant, par exemple, mon bras droit, je serais un être très différent - et à la limite humainement inin-telligible - si je ne me souvenais pas de cette blessure et de son auteur pour le restant de 
mes jours. La blessure, en effet, est un fait constitutif de ma vie, de mon histoire. Des blessures ont été infligées aux peuples d'Afrique du Sud qui sont des faits de leur histoire et que, étant humains, ils ont peu de chance d'oublier ; et, aussi longtemps que nous croyons important de nous rappeler les faits de notre histoire, ce sont des choses qui ne devraient pas être oubliées. L'oubli a cependant également un autre sens, qui se rattache aux enjeux de la réconciliation et du pardon que j'ai abordés dans ma réponse à la deuxième question. Je pense à l'usage qui se traduit quand nous disons à quelqu'un qui nous a blessé : «oublions ça!». Cette forme d'ou-bli n'est pas, et ne peut pas être, un simple effacement d'un fait ou d'un ensemble de faits. Il s'agit plutôt d'une permission, d'une atténuation ou d'un remède qui rompt les liens qui at-ta-chent les auteurs d'un crime à ceux qu'ils ont blessés. Il me serait intolérable que cette forme particulière d'oubli soit impossible, puisque c'est alors tout simplement le pardon qui serait impossible.

\section{NOTES}

1. Anthony Holiday est philosophe, School of Government, University of the Western Cape, (Afrique du Sud). Cet entretien a été réalisé et traduit par John Crowley (novembre 2000).

2. Anthony Holiday, « Forgiving and Forgetting : the Truth and Reconciliation Commission ", in Sarah Nuttall \& Carli Coetzee (eds), The Making of Memory in South Africa, Cape Town, Oxford University Press, 1998, pp. 43-56.

3. Épouse du dirigeant communiste Joe Slovo (NDLR).

\section{INDEX}

Index géographique : Afrique australe, Afrique du Sud (R.S.A.)

Mots-clés : sortie de conflits, Théorie politique, entretien 